\title{
Weak antiferromagnetism and dimer order in quantum systems of coupled tetrahedra
}

\author{
Valeri N. Kotov, ${ }^{1, *}$ Michael E. Zhitomirsky, ${ }^{2}$ Maged Elhajal, ${ }^{1}$ and Frédéric Mila ${ }^{1}$ \\ ${ }^{1}$ Institute of Theoretical Physics, Swiss Federal Institute of Technology (EPFL), 1015 Lausanne, Switzerland \\ ${ }^{2}$ Commissariat á l'Energie Atomique, DSM/DRFMC/SPSMS, 17 avenue des Martyrs, 38054 Grenoble, France
}

(Received 16 September 2004; published 2 December 2004)

\begin{abstract}
We analyze the phases of an $S=1 / 2$ spin model on a lattice of coupled tetrahedra. The presence of both Heisenberg and antisymmetric, Dzyaloshinsky-Moriya interactions can lead to two types of symmetry-broken states: nonmagnetic dimer order and, unexpectedly, exotic four-sublattice weak antiferromagnetic order-a state with a generically small ordered moment and nonzero chirality. The external magnetic field also induces weak antiferromagnetism coexisting with strong dimer correlations in the ground state. These states are formed as a result of broken Ising symmetries and exhibit a number of unusual properties.
\end{abstract}

DOI: 10.1103/PhysRevB.70.214401

PACS number(s): 75.10.Jm, 75.30.Et

\section{INTRODUCTION}

Some of the most challenging and exciting problems in modern solid-state physics are related to the nature of symmetry-broken states and the competition between different types of order in insulating and doped antiferromagnets. ${ }^{1,2}$ There exist numerous materials, ranging from the high-temperature superconductors to molecularbased magnets, that provide a continuous source of inspiration for this research.

In this paper we study the types of order that can occur in quantum spin systems on lattices formed by coupled tetrahedra. Perhaps the most well-known (and still not fully understood) model of this type is the three-dimensional (3D) pyrochlore lattice, composed of corner-sharing tetrahedra. ${ }^{3}$ The purpose of this work, however, is to look at a class of models where the tetrahedra are connected weakly and in a more regular fashion, almost in a 2D square latticelike arrangement. There are two main reasons for this. First, such models allow for more reliable and complete theoretical treatments. Second, we have had in mind potential applications to the $S=1 / 2$ material $\mathrm{Cu}_{2} \mathrm{Te}_{2} \mathrm{O}_{5} \mathrm{Br}_{2}$, ${ }^{4}$ which is representative of such a geometry. Rather unusually, in this material it has been observed that low-energy singlet excitations (measured in Raman spectroscopy) coexist with some kind of (possibly weak) magnetic order, the origin of which is still controversial. ${ }^{4,5}$

We suggest that to understand the properties of the above and similar systems, it is important to take into account antisymmetric, Dzyaloshinsky-Moriya (DM) spin-spin interactions, which are expected to be present in a tetrahedron on symmetry grounds. Our main result, which is quite general and model dependent only in the details, is that under certain conditions the low-energy singlet dynamics can coexist with weak antiferromagnetism, induced by the DM interactions. It is well known that weak magnetic moments can appear near a magnetic-paramagnetic transition boundary or in dimer (gapped) systems in the presence of both DM interactions and an external magnetic field. ${ }^{6}$ In the present work we describe a mechanism for weak antiferromagnetic order, which is induced by the DM interactions even without external fields. We show below that such an exotic possibility exists in tetrahedral systems due to the degeneracy of the ground state on a single tetrahedron. The typical excitation signatures are different from those of conventional spin waves and various nontrivial effects in an external magnetic field, such as magnetic-field-induced order, are also present.

We start with the following spin-1/2 Hamiltonian, which involves both Heisenberg and antisymmetric exchanges:

$$
\hat{\mathcal{H}}=\sum_{i, j} J_{i, j} S_{i} \cdot S_{j}+\sum_{i, j} D_{i, j} \cdot\left(S_{i} \times S_{j}\right),
$$

where the couplings $J_{i, j}$ are distributed as shown in Fig. 1(b). The tetrahedra are represented as plaquettes in Fig. 1(a) and are assumed to be coupled weakly-i.e., $0 \leqslant j_{1}, j_{2} \ll J$, where $J$ is the exchange inside one tetrahedron. All couplings are antiferromagnetic. We analyze the model in two dimensions but the inclusion of three-dimensional couplings is straightforward and does not affect our main results. For simplicity of analysis and notation we set for now $j_{2}=0$, and the effect of $j_{2}$ on our results will be discussed later. The DM couplings have relativistic origin ${ }^{7,8}$ and are expected to be weak, usually at most several percent of the Heisenberg exchange, $\left|\boldsymbol{D}_{i j}\right| \ll J$. The distribution of the couplings $\boldsymbol{D}_{i, j}$ is very lattice-specific, as the presence of the DM interaction on a particular bond is determined by the symmetry of the environment. We will make the assumption that the $\boldsymbol{D}_{i, j}$ 's are present only on the tetrahedra (where the Heisenberg exchange is also dominant) and are nonzero on every bond within one tetrahedron. The distribution of the six vectors $\boldsymbol{D}_{i, j}$, written with respect to the principal axes of the cube in Fig. 1(a) and consistent with the tetrahedral group, is $\boldsymbol{D}_{13}$ $=(D / \sqrt{2})(-1,1,0), \boldsymbol{D}_{24}=(D / \sqrt{2})(-1,-1,0), \boldsymbol{D}_{43}=(D / \sqrt{2})(0$,

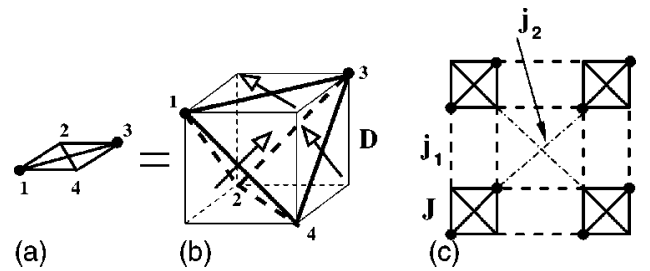

FIG. 1. (a) A tetrahedron with DM vectors represented by arrows (three of the six shown). (b) Two-dimensional lattice of coupled tetrahedra. 
$-1,1), \boldsymbol{D}_{12}=(D / \sqrt{2})(0,-1,-1), \boldsymbol{D}_{14}=(D / \sqrt{2})(1,0,1), \quad$ and $\boldsymbol{D}_{23}=(D / \sqrt{2})(1,0,-1)$. Here $D$ is the magnitude of the DM vectors.

The rest of the paper is organized as follows. In Sec. II we derive the effective Hamiltonian in the weak-coupling limit. The quantum phases of the model are then analyzed in Secs. III and IV. Section V contains our conclusions.

\section{EFFECTIVE HAMILTONIAN IN THE PRESENCE OF DM INTERACTIONS}

First, we describe the states of one tetrahedron, Fig. 1(a). For $D=0$ the ground state is twofold degenerate (with energy $\left.E_{0}=-3 J / 2\right)$ and is in the singlet sector $S^{\text {tot }}=0$. The two ground states are $\left|s_{1}\right\rangle=(1 / \sqrt{3})\{[1,2][3,4]+[2,3][4,1]\}$ and $\left|s_{2}\right\rangle=\{[1,2][3,4]-[2,3][4,1]\}$, where $[k, l]$ denotes a singlet formed by the nearest-neighbor spins $k$ and $l$. The DM interactions break the continuous spin rotational invariance, leading to an admixture of triplets to the ground state. ${ }^{9}$ Introducing the three excited (energy $E_{1}=-J / 2$ ) $S=1$ triplet states $p_{\mu}, q_{\mu}, t_{\mu}, \mu=x, y, z$, in the notation of Ref. 10, we obtain the two new ground states, in the limit $D / J \ll 1$ :

$$
\begin{gathered}
|\Phi\rangle=\left|s_{1}\right\rangle+\frac{3 i D}{2 \sqrt{6} J}\left[\left|p_{x}\right\rangle-\left|p_{y}\right\rangle+\left|q_{x}\right\rangle+\left|q_{y}\right\rangle\right], \\
|\Psi\rangle=\left|s_{2}\right\rangle+\frac{i D}{2 \sqrt{2} J}\left[\left|p_{x}\right\rangle+\left|p_{y}\right\rangle+\left|q_{x}\right\rangle-\left|q_{y}\right\rangle\right]+i \frac{D}{J}\left|t_{z}\right\rangle .
\end{gathered}
$$

These states remain degenerate with $E_{0}^{D M}=-3 J / 2$ $-3 D^{2} /(2 J)$, and their wave functions transform according to the $e_{g}$ irreducible representation of the tetrahedral point group. The diagonal matrix elements of on-site spins between the modified ground states (2) are zero, whereas the off-diagonal matrix elements acquire finite imaginary values $\left\langle\Psi\left|S_{\alpha, n}\right| \Phi\right\rangle= \pm i D / \sqrt{6} J$.

The low-energy sector of the Hilbert space has dimensionality $2^{N_{\text {tet }}}$, where $N_{\text {tet }}$ is the number of spin tetrahedra. The remaining degeneracy is lifted by the intertetrahedral couplings, and we proceed to analyze the nature of the new ground state. For this purpose we introduce a pseudospin $\boldsymbol{T}=1 / 2$ representation, so that $T_{z}=1 / 2$ corresponds to $|\Phi\rangle$ and $T_{z}=-1 / 2$ corresponds to $|\Psi\rangle$. We then obtain the effective Hamiltonian in the ground-state subspace:

$$
\begin{aligned}
\hat{\mathcal{H}}_{\text {eff }}= & -\sum_{\langle i, j\rangle}\left[\Omega_{x} T_{x, i} T_{x, j}+\Omega_{z} T_{z, i} T_{z, j}+\Omega_{y} T_{y, i} T_{y, j}\right. \\
& \left.+\Omega_{x z}^{(i, j)}\left(T_{z, i} T_{x, j}+T_{x, i} T_{z, j}\right)\right]-h \sum_{i} T_{z, i} .
\end{aligned}
$$

Now the site indices $i, \boldsymbol{j}$ refer to the positions of the tetrahedra, and the summation is over nearest neighbors on a square lattice. To lowest order in $j_{1}, D$ the couplings in the different pseudospin directions are explicitly

$$
\Omega_{x}=\frac{j_{1}^{2}}{4 J}, \quad \Omega_{y}=j_{1} \frac{4 D^{2}}{3 J^{2}}, \quad \Omega_{z}=\frac{\Omega_{x}}{3}, \quad h=\frac{2}{3} \Omega_{x} .
$$

The mixed coupling depends on the bond direction $\Omega_{x z}^{(i, j)}$ $=\Omega_{x} e^{i \boldsymbol{Q} \cdot(i-j)} / \sqrt{3}, \boldsymbol{Q}=(\pi, 0)$.

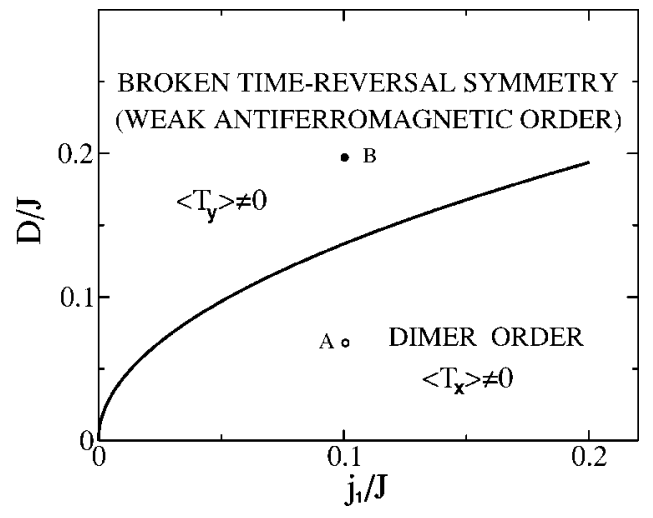

FIG. 2. Phase diagram of the pseudospin Hamiltonian at zero temperature.

The effective Hamiltonian describes an anisotropic ferromagnet in an effective magnetic field $h$ applied along the "hard" $z$ axis. If the field $h$ does not exceed a critical value $h_{c}$ [which we find to be the physical case, corresponding to Eq. (4)], the system breaks Ising symmetry along the soft $x$ or $y$ axes. The selected direction in the $x y$ plane depends on $\Omega_{x} / \Omega_{y}$. The first-order transition line $\Omega_{x}=\Omega_{y}$, on the meanfield level, separates regions with $\left\langle T_{x, i}\right\rangle \neq 0,\left\langle T_{y, i}\right\rangle=0$ (for $\Omega_{x}>\Omega_{y}$ ) and $\left\langle T_{y, i}\right\rangle \neq 0,\left\langle T_{x, i}\right\rangle=0$ (for $\Omega_{y}>\Omega_{x}$ ). The two regions are shown in Fig. 2 as a function of the microscopic parameters. Since $h \neq 0$, throughout the phase diagram $\left\langle T_{z, i}\right\rangle \neq 0$. We now discuss in more detail the nature of symmetry breaking in the two phases.

\section{DIMER PHASE AND MAGNETIC-FIELD-INDUCED ORDER}

The phase with $\left\langle T_{x, i}\right\rangle \neq 0$ corresponds to dimer order. The existence of such a phase for the case $D=0$ was pointed out in Ref. 11. Physically, this means that the ground state of the system is the linear combination $(\sqrt{3} / 2)|\Phi\rangle \pm \frac{1}{2}|\Psi\rangle$, on every site, which is equivalent to $\left\langle T_{x, i}\right\rangle= \pm \sqrt{3} / 4$ and $\left\langle T_{z, i}\right\rangle=1 / 4$ [these values correspond to Eqs. (4)]. The ground state is a real combination of $|\Phi\rangle$ and $|\Psi\rangle$, and therefore the expectation values of local spins vanish, and only dimer order is present in this case, as shown in Fig. 3(a). Since the $\Omega_{x}$ coupling in Eq. (3) has a ferromagnetic sign, the dimer pattern repeats itself on all tetrahedra. Magnetoelastic couplings

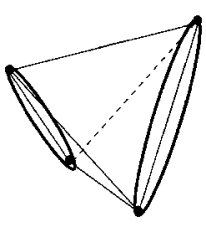

(a)

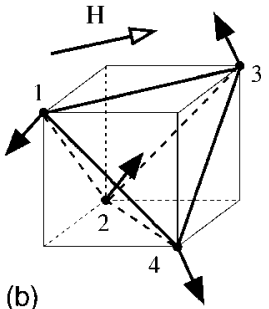

(b)

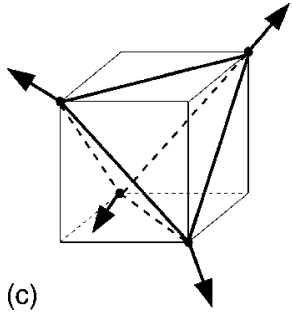

(c)
FIG. 3. (a) Dimer state $\left\langle T_{x, i}\right\rangle \neq 0$. (b) Magnetic moments in an external magnetic field along the 1-3 bond in the dimer phase from (a) (tilt of spins in the field direction not shown). (c) Spin arrangement in the phase $\left\langle T_{y, i}\right\rangle \neq 0$, at zero field. 


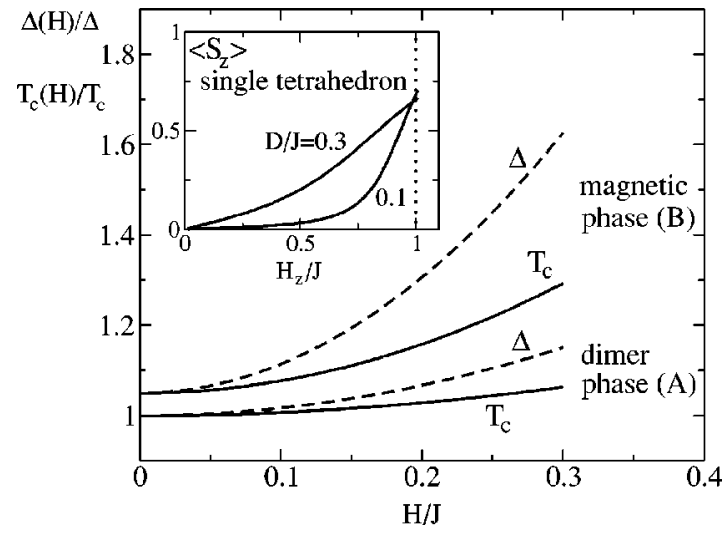

FIG. 4. Dependence of the gap (dashed lines) and $T_{c}$ (solid lines) on magnetic field [applied as in Fig. 3(b)] at points $A$ and $B$ of Fig. 2. Two upper curves shifted by 0.05 for clarity. Inset: magnetic moment $\left\langle S_{z}^{\text {tot }}\right\rangle$ in a magnetic field in the $z$ direction for a single tetrahedron (results identical in the $x, y$ directions). For $D$ $=0,\left\langle S_{z}^{\mathrm{tot}}\right\rangle=0, H_{z} / J<1$.

could also contribute to the dimerization tendency as discussed for the pyrochlore case, ${ }^{12}$ but in our scenario dimerization occurs spontaneously and is due to purely Heisenberg exchanges.

The dimer-ordered state breaks the discrete, Ising symmetry $T_{x, i} \rightarrow-T_{x, i}$ of Eq. (3), and thus the excitation spectrum $\omega(\boldsymbol{k})$ has a gap $\Delta=\omega(\boldsymbol{k}=0)=1.43 \Omega_{x}$ (for $D=0$ ). At finite temperature $T$, the ordered state exists below the critical temperature $T_{c}=0.92 \Omega_{x}$. We have used linear spin-wave expansion and mean-field equations to calculate these quantities.

Due to the presence of triplets in the ground state (2), a (real) external uniform magnetic field $-g \mu_{B} \boldsymbol{H} \cdot \boldsymbol{S}^{\text {tot }}$ also leads to nontrivial effects. On a single tetrahedron $\left(j_{1}=0\right)$ the field produces a finite magnetization in the field direction, shown in the inset of Fig. 4. For small fields $H / J \ll 1$, the contribution is linear and proportional to $(D / J)^{2}(H / J)$. We find that the magnetization is only weakly dependent on the field direction. For $j_{1} \neq 0$, the corrections to the effective Hamiltonian (3) have to be derived, and we find them to be strongly dependent on the field direction. For example, for a weak field in the $x-y$ direction $\boldsymbol{H}=(H / \sqrt{2})(1,1,0)$, Eq. (2) changes to

$$
\begin{gathered}
|\Phi\rangle_{H}=|\Phi\rangle-\frac{\sqrt{3} D H}{2 J^{2}}\left|p_{z}\right\rangle, \\
|\Psi\rangle_{H}=|\Psi\rangle-\frac{D H}{2 J^{2}}\left[\left|q_{z}\right\rangle+\sqrt{2}\left(\left|t_{y}\right\rangle-\left|t_{x}\right\rangle\right)\right] .
\end{gathered}
$$

The energy difference between these states leads to a modification of the "effective field" $h \rightarrow h-D^{2} H^{2} /\left(2 J^{3}\right)$ in Eq. (3). Even though several additional terms are generated in $\hat{\mathcal{H}}_{\text {eff }}$, for $j_{1}, D, H \ll J$, the above effect is dominant. We have recalculated the gap $\Delta(H)$ and the critical temperature $T_{c}(H)$ in the presence of the field and the results are summarized in Fig. 4, where we have plotted these quantities relative to their zero-field values. The gap generally shows a stronger field dependence. We note that the increase as a function of field is not universal, and in fact if a field is applied in the $z$ direction $\boldsymbol{H}=(0,0, H)$, then $\Delta(H)$ and $T_{c}(H)$ would decrease at almost the same rate.

An applied magnetic field also generates staggered antiferromagnetic moments in the plane perpendicular to the field. The corresponding pattern is shown in Fig. 3(b), where $\left\langle\boldsymbol{S}_{3}\right\rangle=-\left\langle\boldsymbol{S}_{4}\right\rangle,\left\langle\boldsymbol{S}_{1}\right\rangle=-\left\langle\boldsymbol{S}_{2}\right\rangle$, and the spins on sites 2 and 4 point along the diagonals of the cube (towards and out of the cube's center, respectively). The value of the magnetic moments in this pattern, repeated on all tetrahedra, is

$$
\left|\left\langle\boldsymbol{S}_{n}\right\rangle\right|=\frac{D H}{J^{2}}\left\langle T_{x, i}\right\rangle=\frac{\sqrt{3} D H}{4 J^{2}}, \quad n=1,2,3,4 .
$$

Field-induced antiferromagnetic ordering is a general feature of singlet systems with DM interactions.

\section{BROKEN TIME-REVERSAL SYMMETRY STATE WITH WEAK ANTIFERROMAGNETIC ORDER}

The phase with $\left\langle T_{y, i}\right\rangle \neq 0$ in Fig. 2 corresponds to a ground state which is a complex linear combination on a single tetrahedron $\alpha|\Phi\rangle \pm i \beta|\Psi\rangle$, where $\alpha, \beta$ are real coefficients. This combination is ferromagnetically repeated on every tetrahedron. Depending on the values of the microscopic parameters, $\sqrt{3} / 4 \leqslant\left|\left\langle T_{y, i}\right\rangle\right| \leqslant 1 / 2$. The resulting state resembles to a large extent a spin-liquid state with a broken time-reversal symmetry proposed some time ago ${ }^{13}$ and characterized by finite scalar chirality $\chi=\left\langle\boldsymbol{S}_{1} \cdot\left(\boldsymbol{S}_{2} \times \boldsymbol{S}_{3}\right)\right\rangle \neq 0$. In our case such a state appears, however, due to the anisotropic DM interaction in a system without spin-rotational invariance, and we have $\chi \sim\left\langle T_{y, i}\right\rangle$. Therefore, a broken timereversal symmetry immediately induces finite antiferromagnetic moments with magnitude

$$
\left|\left\langle S_{n}\right\rangle\right|=\frac{D}{J} \sqrt{2}\left\langle T_{y, i}\right\rangle .
$$

The spins are at an angle $\phi=109.47^{\circ}$ with respect to each other and form a four-sublattice antiferromagnetic structure, as shown in Fig. 3(c). On each tetrahedron the total magnetic moment $\Sigma_{n}\left\langle S_{n}\right\rangle=0$.

Similar to the dimer phase, the broken Ising symmetry leads to an excitation gap and a finite Ising transition temperature, whose values, for dominant DM interactions, are, respectively, $\Delta \simeq \Omega_{y}$ and $T_{c} \simeq \Omega_{y}$. Thus, we find a quite unusual situation: the specific heat exhibits a large anomaly at the transition temperature, where a macroscopic part of the low-temperature entropy $N_{\text {tet }} \ln 2$ freezes out. However, if looked at in neutron experiments such a transition is characterized by development of small antiferromagnetic moments below $T_{c}$. This exotic behavior is due to the fact that the transition itself is driven by low-energy singlet degrees of freedom. In applied magnetic field $\boldsymbol{H}=(H / \sqrt{2})(1,1,0)$, the gap and $T_{c}$ in the broken time-reversal symmetry state scale as shown in Fig. 4, upper set of curves. We find that generally the field dependence is stronger than in the dimer phase, which is mainly due to the presence of the DM scale $D$ in the two observables without a field. 
We note that $\Omega_{y}$ depends linearly on $j_{1}$ while $\Omega_{x}$ is quadratic, see Eq. (4). Thus, even though the $T_{y}$ (magnetic) and the $T_{x}$ (dimer) orders compete with each other, at sufficiently small $j_{1}$ the $T_{y}$ order is expected to dominate (although only in an asymptotic sense for the present model). The tendency towards $T_{y}$ order is further enhanced by the presence of $j_{2} \neq 0$ [see Fig. 1(b)]. This coupling frustrates the $T_{x}$ component while strengthening $T_{y}$, which results in a shift of the phase boundary in Fig. 2 downwards. If we also consider a situation when $j_{2}$ is the dominant intertetrahedral exchange, $J \gg j_{2} \gg j_{1}$, and set $j_{1}=0$, then we find that $T_{x}$ couplings are generated only in fourth order, $\Omega_{x} \sim j_{2}^{4} / J^{3}$. This again means that the $T_{y}$ order can occur under much easier conditions. Nonetheless, only on lattices that have the tendency to produce true spin-liquid ground states do we expect the $T_{y}$ order to occur spontaneously. Good candidates appear to be the pyrochlore lattice $^{3}$ as well as the kagomé lattice. ${ }^{14}$ DMinduced ordering has been discussed in the latter case from a quasiclassical perspective. ${ }^{15}$

\section{DISCUSSION AND CONCLUSIONS}

Our calculations have been performed for ideal tetrahedra while weak distortions (such as asymmetry in the Heisenberg exchanges or deviations of the DM vectors from the tetrahedral symmetry) would introduce a small gap on a single tetrahedron. However, as long as $j_{1}, j_{2}$ are sufficiently large, relative to this gap, the basic characteristics of the phases described above will remain unchanged. The DM order may deviate from Fig. 3(c), while preserving the property $\Sigma_{n}\left\langle\boldsymbol{S}_{n}\right\rangle=0$.

The physics described in the present work occurs on the small energy scales $\Omega_{x}, \Omega_{y} \ll J$, while upon increasing the intertetrahedral couplings to $j_{1}, j_{2} \sim J$, a transition to a more conventional Néel phase is expected to take place. ${ }^{5,16}$ The exotic phases we have found are certainly stable as long as the system is sufficiently far from the Néel order; i.e., the triplet gap is nonzero and all relevant dynamics is governed by the singlet sector [or the mixed states Eq. (2) in the presence of DM interactions] and, therefore, can be described by the effective Hamiltonian, Eq. (3). We have made sure that this condition is fulfilled by using the weak-coupling analysis, although our results are expected to be qualitatively valid also for moderately large $j_{1}, j_{2}$ subject, as already mentioned, to the condition that the triplet gap is sizable enough. Thus, on general grounds we expect a transition as a function of $j_{1}$ or $j_{2}$ between phases with exotic (weak magnetic) order, discussed in this work, and the Néel-ordered state, although the exact determination of the transition boundary is a difficult problem and has not been the subject of this work. Instead, we have concentrated on the "universal" properties of the DM-induced exotic phases which are clearly present in the weak-coupling limit and accurately described by a Hamiltonian of the form of Eq. (3).

The states we have found at low energies have quite unusual characteristics, which we will now summarize. (i) Due to the presence of the DM interactions, the spin-rotational invariance is broken and the ground state develops a small magnetic moment in a field, with the uniform magnetization showing a characteristic linear behavior in weak magnetic fields [Fig. 4 (inset)]. (ii) Either dimerized or four-sublattice, weakly antiferromagnetic states emerge as the ground state of the system. Both exhibit gaps in the spectrum since only Ising symmetries are broken. (iii) The dominant components in the wave functions, Eq. (2), are singlets; thus, large spectral weight $S=0$ transitions at low energy (equal to the gap $\sim \Omega_{x}, \Omega_{y}$ ) are present in the Raman spectrum. (iv) Both the gap and $T_{c}$ depend on magnetic field with a strong directional dependence. (v) The dominant $S=1$ excitation has a large gap of order $J \gg \Omega_{x}, \Omega_{y}$.

We now comment on possible applications of our theory to $\mathrm{Cu}_{2} \mathrm{Te}_{2} \mathrm{O}_{5} \mathrm{Br}_{2}$. The properties of this material are indeed consistent with signatures (i) - (iv) above, in particular the compound exhibits $^{4,5}$ (a) a weak magnetization in a field, (b) a sharp zero-field, low-energy Raman peak ("singlet gap" $\Delta \approx 24 \mathrm{~K}$ ), disappearing at $T_{c} \approx 11 \mathrm{~K}$, and (c) a magnetic field dependence (increase) of both $\Delta$ and $T_{c}$. Experimentally the most important unanswered question remains whether the ground state below $T_{c}$ is magnetically ordered or not, with recent NMR experiments pointing to some kind of magnetic order. ${ }^{17}$ This would suggest, in our scenario, that the ground state is of the type shown in Fig. 3(c). The crucial test for our ideas, and thus our prediction, would be the observation of a large triplet gap (larger than the singlet gap of $24 \mathrm{~K}$ ) in the neutron spectrum [point (v) above]. The microscopic values of the exchanges are still rather controversial ${ }^{18}$ and consequently we have not attempted detailed fits but have used, phenomenologically, the model of Fig. 1. We emphasize that in our model a weak magnetic moment is a generic feature of the $\left\langle T_{y, i}\right\rangle \neq 0$ phase (and the dimer phase in magnetic field) and does not require a fine-tuning of parameters to achieve proximity to a magnetic-paramagnetic boundary, as in the purely Heisenberg case. ${ }^{5}$

Finally, on a purely fundamental level, we have found an unconventional mechanism for weak antiferromagnetism. Our results are to be contrasted with the usual effect the DM interaction produces ${ }^{7,8}$ — namely, weak ferromagnetism in an otherwise antiferromagnetic system. In the present work we have shown how weak antiferromagnetic order can emerge from a singlet background.

\section{ACKNOWLEDGMENTS}

We are grateful to C. Lhuillier, C. Lacroix, O. Sushkov, and C. Berthier for stimulating discussions. This work was supported by MaNEP and the Swiss National Fund. 
*Electronic address: valeri.kotov@epfl.ch

${ }^{1}$ S. Sachdev, Rev. Mod. Phys. 75, 913 (2003).

${ }^{2}$ G. Misguich and C. Lhuillier, in Frustrated Spin Systems, edited by H. T. Diep (World Scientific, Singapore, 2003), p. 1; condmat/0310405 (unpublished), and cited references.

${ }^{3}$ A. B. Harris, A. J. Berlinsky, and C. Bruder, J. Appl. Phys. 69, 5200 (1991); H. Tsunetsugu, Phys. Rev. B 65, 024415 (2002).

${ }^{4}$ P. Lemmens, K.-Y. Choi, E. E. Kaul, Ch. Geibel, K. Becker, W. Brenig, R. Valenti, C. Gros, M. Johnsson, P. Millet, and F. Mila, Phys. Rev. Lett. 87, 227201 (2001).

${ }^{5}$ C. Gros, P. Lemmens, M. Vojta, R. Valenti, K.-Y. Choi, H. Kageyama, Z. Hiroi, N. V. Mushnikov, T. Goto, M. Johnsson, and P. Millet, Phys. Rev. B 67, 174405 (2003).

${ }^{6}$ S. Miyahara, F. Mila, K. Kodama, M. Takigawa, M. Horvatic, C. Berthier, H. Kageyama, and Y. Ueda, J. Phys.: Condens. Matter 16, S911 (2004).

${ }^{7}$ I. Dzyaloshinsky, J. Phys. Chem. Solids 4, 241 (1958).

${ }^{8}$ T. Moriya, Phys. Rev. 120, 91 (1960).

${ }^{9}$ The $S=2$ excited states do not mix with the ground state.
${ }^{10}$ M. E. Zhitomirsky and K. Ueda, Phys. Rev. B 54, 9007 (1996).

${ }^{11}$ V. N. Kotov, M. E. Zhitomirsky, and O. P. Sushkov, Phys. Rev. B 63, 064412 (2001).

${ }^{12}$ Y. Yamashita and K. Ueda, Phys. Rev. Lett. 85, 4960 (2000); O. Tchernyshyov, R. Moessner, and S. L. Sondhi, Phys. Rev. B 66, 064403 (2002).

${ }^{13}$ X. G. Wen, F. Wilczek, and A. Zee, Phys. Rev. B 39, 11413 (1989).

${ }^{14}$ C. Waldtmann, H. U. Everts, B. Bernu, P. Sindzingre, C. Lhuillier, P. Lecheminant, and L. Pierre, Eur. Phys. J. B 2, 501 (1998); F. Mila, Phys. Rev. Lett. 81, 2356 (1998).

${ }^{15}$ M. Elhajal, B. Canals, and C. Lacroix, Phys. Rev. B 66, 014422 (2002).

${ }^{16}$ W. Brenig, Phys. Rev. B 67, 064402 (2003).

${ }^{17} \mathrm{C}$. Berthier (private communication).

${ }^{18}$ M. Johnsson, K. W. Tornroos, F. Mila, and P. Millet, Chem. Mater. 12, 2853 (2000); R. Valenti, T. Saha-Dasgupta, C. Gros, and H. Rosner, Phys. Rev. B 67, 245110 (2003); V. V. Mazurenko and V. I. Anisimov (unpublished). 